\title{
More evidence is needed to show that heritability and selection are not associated
}

\author{
Jon E. Brommer (1)
}

ARISING FROM J. J. C. Ramakers, A. Culina, M. E. Visser \& P. Gienapp Nature Ecology \& Evolution https://doi.org/10.1038/s41559-018-0577-4 (2018)

Under genotype-environment interactions (GEI) the potential response to selection on trait $Y$ is environment specific, and also the strength of selection on $Y$ may interact with the environment (SEI). Ramakers et al. ${ }^{1}$ quantified GEI and SEI and their covariation by analysing data on 50 traits from 16 wild populations obtained from public data repositories. In doing so, a challenge is that the true environmental driver $E$ is latent (and hence unknown) (Fig. 1). Evolutionary quantitative genetic research typically ${ }^{2}$ describes true $E$ by selecting the proxy environmental variable $\mathrm{pr} E$ that shows the maximal absolute correlation to the plastic response of trait $Y$. In contrast, Ramakers et al. used the trait mean $\bar{Y}$ as a proxy for $E$. As a result of attenuation $^{3}$, all approaches to proxy $E$ bias the effect sizes of plasticity, GEI and SEI towards zero, as they introduce measurement error in the explanatory variable $E$. The severity of this bias towards zero increases when the correlation of proxy environment with true environment $\left(r_{E-\bar{Y}}\right.$ and $r_{\bar{Y}-\text { prE }}$ in Fig. 1), termed reliability, decreases ${ }^{3,4}$.

Ramakers et al. concluded that the processes of GEI and SEI are not coupled in nature, as only $12 \%$ of traits showed evidence of such coupling. Such claims, however, demand that the inferences of GEI and SEI are the strongest possible, which-because of attenuationholds only if $r_{E-\bar{Y}}$, the reliability of $\bar{Y}$, exceeds the reliability of the best environmental proxy (that is, $\left|r_{E-\bar{Y}}\right|>\left|r_{E-\mathrm{pr} E}\right|$ ) for each trait studied. Importantly, $r_{E-\bar{Y}}$ is fixed given that data were collected and trait values were expressed as they were. In contrast, the usual procedure of maximizing the correlation between proxy environment and focal trait implicitly maximizes the reliability of the proxy environment, because $\max \left\{\left|r_{\bar{Y}-\mathrm{pr} E}\right|\right\}=\max \left\{\left|r_{E-\bar{Y}} r_{E-\mathrm{pr} E}\right|\right\} \propto \max \left\{\left|r_{E-\mathrm{pr} E}\right|\right\}$. As a consequence, Ramakers et al. make the strong a priori assumption that using the mean trait value is the best description of true $E$ in each system studied, without considering alternative proxies. Suppose, for example, that 'spring environment' is the true $E$ and affects between-year variation in mean trait values $\left(r_{E-\bar{Y}}=0.6\right)$, but the average temperature in two weeks in April, which is also affected by $E$, correlates to annual trait means $\left|r_{\bar{Y}-\text { prE }}\right|=0.42$. From the path diagram (Fig. 1), it follows that the reliability of this environmental proxy $\left|r_{E-\mathrm{pr} E}\right|=0.7$, which exceeds the reliability of $\bar{Y}$. For the traits considered by Ramakers et al., it remains possible that GEI and SEI are associated, but their inferred effect sizes (and hence their association) are, for most traits, severely underestimated because the reliability of $\bar{Y}$ is below that of another proxy for true $E$. If this scenario is true, repeating analyses using an environmental proxy with the highest correlation to $\bar{Y}$ would be expected to increase inferred effect sizes of GEI, which may alter the inferences of Ramakers et al. An alternative approach to overcome attenuation is to load multiple proxy environmental variables onto the latent environment ${ }^{5}$. In any case, to make proper inferences on the processes of GEI and SEI and whether these are coupled in nature, it is necessary to quantitatively consider alternative proxies for true $E$.

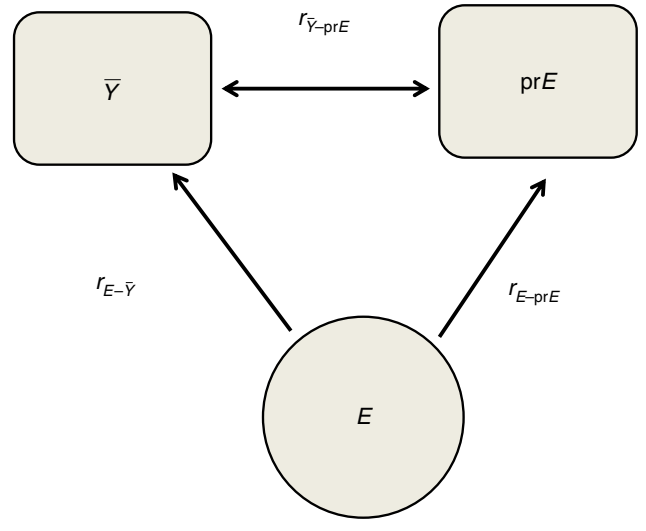

Fig. 1 | Relationships between key parameters. A path diagram of how the latent (unknown) true environment $E$ affects (arrows) the observed trait mean $\bar{Y}$ as well as measurements of the proxy environmental variable $\operatorname{pr} E$, thereby producing covariation (double arrow) between $\bar{Y}$ and $\operatorname{pr} E$. The effect sizes of all paths are denoted as correlation coefficient $r$.

Received: 14 November 2018; Accepted: 20 August 2019; Published online: 23 September 2019

\section{References}

1. Ramakers, J. J. C., Culina, A., Visser, M. E. \& Gienapp, P. Environmental coupling of heritability and selection is rare and of minor evolutionary significance in wild populations. Nat. Ecol. Evol. https://doi.org/10.1038/ s41559-018-0577-4 (2018).

2. van de Pol, M. et al. Identifying the best climatic predictors in ecology and evolution. Methods Ecol. Evol. 7, 1246-1257 (2016).

3. Spearman, C. "General intelligence", objectively determined and measured. Am. J. Psychol. 15, 72-101 (1904).

4. Gienapp, P. The choice of the environmental covariate affects the power to detect variation in reaction norm slopes. Preprint at bioRxiv https://doi. org/10.1101/311217 (2018).

5. Rigdon, E. Demonstrating the effects of unmodeled random measurement error. Struct. Equ. Model. 1, 375-380 (1994).

\section{Acknowledgements}

I thank S. Helle for discussion on attenuation.

\section{Competing interests}

The author declares no competing interests.

\section{Additional information}

Correspondence and requests for materials should be addressed to J.E.B.

Reprints and permissions information is available at www.nature.com/reprints.

Publisher's note Springer Nature remains neutral with regard to jurisdictional claims in published maps and institutional affiliations.

(C) The Author(s), under exclusive licence to Springer Nature Limited 2019 